\title{
Heavy drinking greatly increases the risk of cirrhosis in patients with HCV hepatitis
}

Harris DR, Gonin G, Alter HJ, et al. The relationship of acute transfusion-associated hepatitis to the development of cirrhosis in the presence of alcohol abuse. Ann Intern Med 2001;134:120-4.

\section{Aims}

To quantify the relationship between heavy alcohol intake in conjunction with transfusion associated hepatitis and the subsequent development of cirrhosis.

\section{Design}

Retrospective cohort study of subjects found to have transfusion associated hepatitis.

\section{Setting}

Liver clinics in US university and government hospitals.

\section{Methods}

Retrospective cohort study of 1030 patients with transfusion associated hepatitis in the USA between 1968 and 1980. Included patients from three prospective studies of cases of transfusion related hepatitis C infection with matched controls with similar transfusion histories but who had not developed hepatitis. Data were obtained from case notes, death registries, and personal interview. History of excessive alcohol consumption was obtained from personal interview of patient or proxy. Criteria for heavy consumption were well defined although absolute quantities of alcohol could not be determined.

\section{Results}

A total of $836(81 \%)$ of the original cohort of 1030 were included. Baseline characteristics of included and excluded patients were similar. Comparisons between cases developing cirrhosis $(n=53)$ and those who did not $(n=783)$ found no association with tattooing, occupational exposure, travel to areas where hepatitis $C$ was endemic, or intravenous drug use. Development of cirrhosis was associated with ethnicity, number of units transfused, and history of heavy alcohol consumption. Patients with transfusion associated hepatitis $\mathrm{C}$ were 7.8 times (confidence interval (CI) 4.0-15.1) more likely to develop cirrhosis than controls. A history of heavy alcohol consumption was strongly associated with an increased risk of developing cirrhosis (odds ratio 4.0 (CI 2.1-7.7)). Patients with both transfusion related hepatitis $\mathrm{C}$ and a history of heavy alcohol use were 31.1 times (CI 11.4-84.5) more likely to develop cirrhosis than controls with no history of alcohol abuse or hepatitis C.

\section{Conclusion}

Heavy alcohol abuse greatly exacerbates the risk of cirrhosis among patients with hepatitis $\mathrm{C}$ virus infection. This finding emphasises the need to counsel such patients about their drinking habits.

\section{Comment}

After infection with hepatitis C virus (HCV), approximately $15 \%$ of infected individuals recover spontaneously, a further $25 \%$ have an asymptomatic illness with persistently normal transaminases and benign histological changes, and the remaining $60 \%$ develop biochemical and histological evidence of chronic hepatitis. ${ }^{1}$ In studies with 10-20 years of follow up, progression to cirrhosis has been observed in $2-30 \%$ of patients with chronic hepatitis, with the highest rates reported in post-transfusion cases and the lowest in young women infected with anti-D immune globulin. ${ }^{2}$ Factors increasing the likelihood of disease progression include older age at viral acquisition, human immunodeficiency virus (HIV) or hepatitis B virus (HBV) coinfection, and possibly male sex. ${ }^{1}$ In addition, many studies have shown that excessive alcohol intake is associated with more severe and rapidly progressive disease leading more frequently to cirrhosis and hepatocellular carcinoma. A study immunising mice with HCV core constructs has suggested that this effect is due to alcohol inhibiting the antiviral cellular immune response resulting in enhanced $\mathrm{HCV}$ replication. ${ }^{3}$ While there is no doubt that heavy drinking adversely effects the outcome of chronic $\mathrm{HCV}$, the level of intake that is deleterious and the magnitude of the effect is less clear. Studies that have examined alcohol dose have generally reported intakes of 40-50 g/day as the level at which disease severity and progression rate are increased. ${ }^{45}$ However, in some studies, $10 \mathrm{~g} /$ day has been associated with an adverse outcome in terms of histology and viral load. ${ }^{6}$ This has led to suggestions that patients with HCV should restrict their intake to less than one drink per day, with candidates for antiviral therapy abstaining completely. ${ }^{7}$ However, the key question for both patients and clinicians alike is: "What is the magnitude of the alcohol effect on the progression of HCV?"

This has been addressed by the study from Harris et al which examined the effect of alcohol intake on the outcome of transfusion associated $\mathrm{HCV}$ infection in patients enrolled into three prospective studies of non-A, non-B hepatitis. After a median 15 year follow up, patients with HCV were almost eight times more likely to have developed "clinical" cirrhosis than "matched" controls. In patients with HCV, alcohol abuse was associated with 2-8 times the risk of cirrhosis, increasing the risk over controls to more than 30 . Unfortunately, the study design means that few conclusions can be drawn from the data presented. Two of the three studies specifically excluded alcoholics in their original study design ${ }^{8}$ casting doubt on the classification of alcohol abuse in 591/836 patients. Furthermore, $72 \%$ of cirrhotics and $55 \%$ of non-cirrhotics were deceased. Classification of alcohol abuse was therefore based on interviewing friends and relatives or examining medical records, leading to a gross underestimate of intake. This may explain why only 34/1030 patients had both $\mathrm{HCV}$ and a history of alcohol abuse, which accounts for the large confidence intervals for the reported risk ratios. The only tentative conclusion that can be drawn from the data 
is that very heavy alcohol intake increases the risk of cirrhosis in patients with HCV by approximately fourfold. No conclusions can be drawn on the effect of heavy alcohol intake on the risk of cirrhosis in non-HCV patients as all or virtually all drinkers with cirrhosis had HCV.

Patients with chronic HCV should continue to limit their alcohol intake as much as possible. For the "inquisitive" patient the best estimate of the magnitude of the effect of "moderate" intake on disease outcome comes from a prospective study of over 2000 patients showing that liver fibrosis in patients drinking more than $50 \mathrm{~g} /$ day progresses $34 \%$ faster than in abstainers, shortening their time to cirrhosis by eight years. ${ }^{5}$

C P DAY Centre for Liver Research, Medical School, Framlington Place, Newcastle upon Tyne NE2 4HH, UK c.p.day@ncl.ac.uk
1 Alberti A, Chemello L, Benvegnu L. Natural history of hepatitis C. 7 Hepatol 1999;31(suppl 1):17-24.

2 Kenny-Walsh E, for the Irish Hepatology Research Group. Clinical outcomes after hepatitis $\mathrm{C}$ infection in healthy young adults. $N$ Engl f Med 1999;340:1228-33.

3 Geissler M, Gesein A, Wands JR. Inhibitory effects of chronic ethanol consumption on cellular immune responses to hepatitis $\mathrm{C}$ virus core protein are reversed by genetic immunizations augmented with cytokine expressing plasmids. F Immunol 1997;159:5107-13.

4 Wiley TE, McCarthy M, Breidi L, et al. Impact of alcohol in the progression of liver disease caused by hepatitis C virus infection. Hepatology 1998;28:805-9.

5 Poynard T, Bedossa P, Opolon P. Natural history of liver fibrosis progression in patients with chronic hepatitis C. Lancet 1997;349:825-32.

6 Cromie SL, Jenkins PJ, Bowden DS, et al. Chronic hepatitis C: effect of alcohol on hepatitic activity and viral titre. F Hepatol 1996;25:821-6.

7 Schiff E. Hepatitis C and alcohol. Hepatology 1997;26(suppl 1):39-42S.

8 Seeff LB, Buskell-Bales Z, Wright EC, et al. Long-term mortality after transfusion-associated non-A, non-B hepatitis. N Engl f Med 1992;327: 1906-11.

\title{
7th European Forum on Quality Improvement in Health Care
}

\author{
21-23 March 2002 \\ Edinburgh, Scotland
}

We are delighted to announce this forthcoming conference in Edinburgh. Delegate enquiries are welcome.

The themes of the Forum are:

- Leadership, culture change, and change management

- Achieving radical improvement by redesigning care

- Health policy for lasting improvement in health care systems

- Patient safety

- Measurement for improvement, learning, and accountability

- Partnership with patients

- Professional quality: the foundation for improvement

- Continuous improvement in education and training

- People and improvement.

Presented to you by the BMJ Publishing Group (London, UK) and Institute for Healthcare Improvement (Boston, USA). For more information contact: quality@bma.org.uk or look at the website www.quality.bmjpg.com. Tel: +44 (0)20 7383 6409; fax: +44 (0)20 73736869 . 